\title{
Efficiency of Optical Sensors with Quasi Gaussian Beam for Determining Cholesterol Concentration
}

\author{
Mohammad Budiyanto ${ }^{1}$ \\ Department of Natural Science \\ Universitas Negeri Surabaya \\ Surabaya, Indonesia \\ ${ }^{1}$ mohammadbudiyanto@unesa.ac.id
}

\author{
Suhariningsih ${ }^{2}$, Moh. Yasin ${ }^{3}$ \\ Department of Physics \\ Airlangga University \\ Surabaya, Indonesia \\ ${ }^{2}$ suhariningsih@fst.unair.ac.id, ${ }^{3}$ yasin@ @st.unair.ac.id
}

\begin{abstract}
Research on fiber optic sensors with Quasi Gaussian beam has been carried out aiming to analyze the beam intensity profile of the light beam, sensor performance and sensor sensitivity to determine cholesterol concentration. The concept of laser beam propagation is guided by an optical fiber bundle in a solution with a cholesterol concentration that varies from 0 to 300 ppm. The detection mechanism of cholesterol concentration is the propagation of the $\mathrm{He}-\mathrm{Ne}$ laser beam with a wavelength of 632.5 $\mathrm{nm}$ through the fiber optic bundle to the cholesterol solution, then reflected using a flat mirror. Reflective waves enter optical fibers through fiber receiving. This signal is captured by a silicon detector (SL-818, Newport) in the form of electrical voltage. The results show that the power profile increases with increasing distance of object shift from optical fiber. After reaching peak output power, the power profil will decrease exponentially with increasing distance. The performance of the shift sensor of the Quasi Gaussian beam optical fiber to determine the concentration of cholesterol achieves high sensitivity, linearity and accuracy with a sensitivity value of $0.08 \mathrm{mV} / \mathrm{ppm}$, linearity of more than $97 \%$, linear range of $0-300 \mathrm{ppm}$, resolution of $1.76 \mathrm{ppm}$ and standard deviation of $0.14 \mathrm{mV}$. Sensitivity through mathematical analysis using the Quasi Gaussian beam approach is higher than the sensitivity of the Gaussian beam. Based on the output power profile, sensor performance, sensitivity through the flat mirror reflection field and sensor stability, it can be concluded that the optical fiber sensor using Quasi Gaussian beam can determine cholesterol concentration.
\end{abstract}

Keywords-optical sensor; Quasi Gaussian beam; sensor performance; concentration cholesterol

\section{INTRODUCTION}

Optical fiber is a waveguide transmission channel that is widely used for telecommunications. Optical fiber is also applied in research using sensors known as Optical Fiber Sensors (OFS). The application of OFS with the principle of optical fiber shift is widely used to detect the concentration of compounds in solution. Detection of concentration using optical fiber shift sensors is widely used to determine the concentration of the solution by various methods such as fiber optic bundle, fiber optic taper, and fiber optic coupler.

OFS applied research of various types of optical fibers continues to develop. Detection of Rhodamine B levels in distilled water based on a shift sensor using fiber coupler and concave mirror [1]. Intensity-based optical fiber sensor for calcium detection [2]. Fiber optic shift sensor for detection of honey purity in distilled water [3]. Multimode design of taper fiber sensors for glucose detection [4]. $\mathrm{NaCl}$ salt detection fiber optic sensor with flat mirror and concave mirror [5]. Optical fiber bundle using a theoretical analysis of the Gaussian Beam approach. The bundle fiber optic shift sensor uses a QuasiGaussian beam approach [6]. Some of these OFS applied articles motivate researchers to conduct OFS research to determine cholesterol concentration in a solution using Quasi-Gaussian beam through mathematical analysis and experimental approaches.

Cholesterol is a complex fat compound produced by the body with various functions. Cholesterol is found in the bloodstream or body cells needed for cell wall formation and as a raw material for several hormones. But if excessive cholesterol levels in the blood, it can lead to diseases, including coronary heart disease and stroke. Normal cholesterol must be below 200 $\mathrm{mg} / \mathrm{dl}$. If it is above $240 \mathrm{mg} / \mathrm{dl}$, it will be at high risk for diseases such as heart attack or stroke [7].

Detection of cholesterol concentrations has been carried out by many researchers such as, Magnetite as a platform material for detecting glucose, ethanol and cholesterol [8]. Amperometric detection of cholesterol uses cobalt (II) chloride as an electrocatalyst in aprotic media [9]. Sensitive and selective electrochemical sensors use silver nanoparticles modified glass carbon electrodes for the determination of bovine serum cholesterol [10]. Electrochemical sensors based on molecular film printing on carbon nanoparticles modified electrodes for cholesterol determination [11]

Based on this description, a fiber optic sensor research will be developed as an instrument to determine cholesterol concentration using a Quasi-Gaussian beam. The general objective in this study is to develop an optical fiber sensor and increase sensor efficiency with a Quasi-Gaussian beam approach to determine cholesterol concentration. Specific objectives in this study were to analyze the intensity profile of the output beam of the optical fiber shift sensor detected by photodetector against cholesterol solution samples [12]. determine the performance achievement of the fiber optic shift sensor using a Quasi Gaussian beam to determine cholesterol concentration. 


\section{EXPERIMENT SETUP}

The research method of determining cholesterol concentration based on laser beam character to cholesterol solution material through optical fiber shift sensor by using flat mirror is a modulation of Laser light intensity. The light beam that enters the optical fiber of the receiver and is processed by an optical detector into an electrical signal to be displayed on the computer. Experimental results will be obtained linieritas relationship between the peak voltage of the sensor output as a function of variation of cholesterol concentration and will be measured paramater and sensor performance include sensitivity, linear range, and linearity.

The research apparatus consists of He-Ne laser (Thorlabs, $632.5 \mathrm{~nm}, 5 \mathrm{~mW}$ ), Fiber optic bundles, 818-SL (Newport) optical detectors, Chopper and Chopper controllers (SR540, Stanford Research System, Inc.), Lock-in amplifier (SR510, Stanford Research System, Inc.), flat and concave mirror, position micrometer (Newport), PC, and other supporting devices. The material used in this study is a standard cholesterol solution consisting of cholesterol soluble substances $\left(\mathrm{C}_{27} \mathrm{H}_{45} \mathrm{OH}\right)$, capric acid $\left(\mathrm{CH}_{3}\left(\mathrm{CH}_{2}\right){ }_{8} \mathrm{COOH}\right)$ and methanol $\left(\mathrm{CH}_{3} \mathrm{OH}\right)$ solvents.

Procedure Research determines cholesterol concentration based on the character of the laser beam on the material of cholesterol solution through a fiber optic shift sensor using a flat mirror is a laser light intensity modulation. A beam of light that enters the receiver's optical fiber and is processed by an optical detector into an electrical signal that will be displayed on the computer. The experimental results will obtain a linearity relationship between the peak voltage of the sensor output as a function of variation in cholesterol concentration and the parameters and performance of sensors will be measured including sensitivity, linear range, and linearity.

The design of the fiber optic sensor determines the concentration of cholesterol using flat mirror reflection fields to determine the sensitivity value approach using a Quasi Gaussian beam as shown in Figure 1.

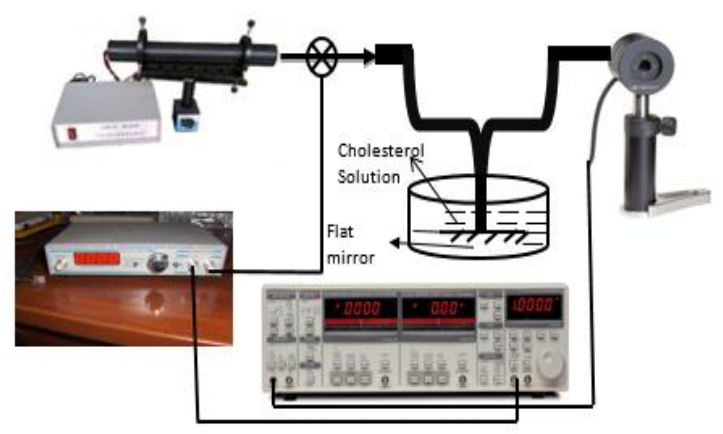

Fig. 1. Experimental setup for optical fiber displacement sensor using flat mirror

The first step is to characterize the shift sensor to determine the shift of the bundle fiber sensing channel to the mirror. The detection starts when the fiber bundle is placed coinciding with a flat and concave mirror that is at the shift of $\mathrm{z}=0$. The fiber bundle is placed on the micrometer position shifted every $50 \mu \mathrm{m}$. At each shift position the detector output voltage is measured so that the data is obtained in the form of detector output voltage as a function of shifting the fiber bundle. Detection was carried out on several variations of concentration including $0 \mathrm{ppm}, 50 \mathrm{ppm}$, $100 \mathrm{ppm}, 150 \mathrm{ppm}, 200 \mathrm{ppm}, 250 \mathrm{ppm}$ and $300 \mathrm{ppm}$.

Furthermore, in the experimental test, the data obtained is in the form of the peak output voltage of the sensor as a function of variation in cholesterol concentration. With this function, the front slope and the back linear area will be determined. Linearity test of front slope area and rear slope is used linear regression test. If it is obtained the value of linear regression coefficient approaching one (more than 0.9), then the two slopes are linear regions, and the area is the sensor working area. Then the peak voltage value is determined as a function of cholesterol concentration. From these results, sensor performance will be obtained which includes resolution, sensitivity, linearity, linear range and standard deviation of fiber optic sensors using a Quasi Gaussian beam.

\section{RESULTS AND DISCUSSIONS}

The results of the study of fiber optic sensors for the determination of cholesterol concentration based on the intensity profile of the detected external light beam are output voltage. Figure 2 shows the detector output voltage as a function of the fiber bundle shift for each concentration from $0 \mathrm{ppm}$ to $300 \mathrm{ppm}$. The figure shows the relationship between changes in cholesterol concentration variation to the maximum output voltage at each concentration. The higher the cholesterol concentration the lower the detector output voltage is measured

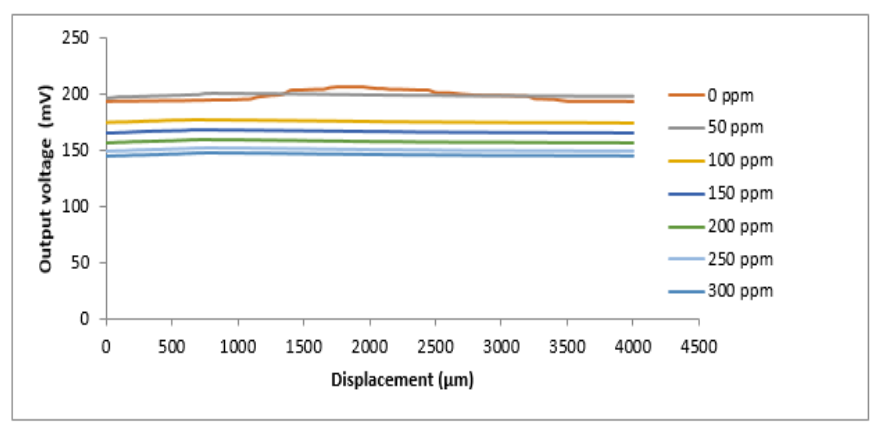

Fig. 2. Output voltage against displacement for various concentration of cholesterol

The detector output voltage detection data starts when the bundle fiber is placed coinciding with the mirror at the shift of $\mathrm{z}$ $=0$. The bundle fiber is placed in the micrometer position shifted every $50 \mu \mathrm{m}$. At each shift position, the detector output voltage is measured to obtain the maximum output voltage of each variation in cholesterol concentration. The maximum output voltage value of the variation in cholesterol concentration as in table 1 .

Table I. Peak Voltage Of The Concentration Of Cholesterol

\begin{tabular}{|c|c|}
\hline Concentration (ppm) & Peak voltage (mV) \\
\hline 0 & 205.2 \\
\hline 50 & 201.0 \\
\hline 100 & 176.8 \\
\hline 150 & 167.5 \\
\hline 200 & 158.6 \\
\hline 250 & 150.1 \\
\hline 300 & 146.3 \\
\hline
\end{tabular}


Figure 3 shows that the maximum output voltage appears to decrease linearly to the increase in the concentration of cholesterol solution

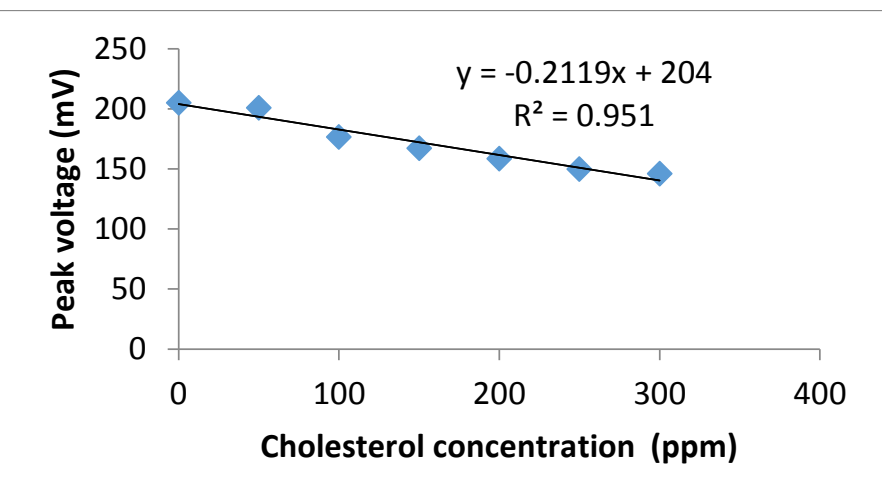

Fig. 3. Peak voltage versus concentration of cholesterol for displacements using flat mirror

The results of the detection analysis showed that the maximum output voltage showed a linear decrease in the concentration of cholesterol solution with a sensitivity of 0.2119 $\mathrm{mV} / \mathrm{ppm}$ and linearity of $95.1 \%$. Based on the experimental results, the determination of cholesterol concentration using quasi Gaussian beam OFS shows that the optical fiber output power profile will increase by increasing the distance of object shift from the optical fiber bundle, after reaching the peak the output power will decrease exponentially with increasing distance in each variation of cholesterol concentration. Figure 2 shows that the concentration of $0 \mathrm{ppm}$ output voltage starts at $194.1 \mathrm{mV}$ at a minimum distance. Increasing the distance to the solution sample affects the increase in the output voltage until it reaches the maximum output voltage of $205.2 \mathrm{mV}$ at a distance of $1850 \mu \mathrm{m}$. In the second sample with a concentration of 50 ppm the minimum output voltage starts at $196.2 \mathrm{mV}$ and increases in voltage by increasing the distance to the sample solution until it reaches maximum output voltage value is 201.0 $\mathrm{mV}$ at a distance of $800 \mu \mathrm{m}$. In the third sample the concentration of $100 \mathrm{ppm}$ output voltage starts $174.8 \mathrm{mV}$ at a minimum distance. Increasing the distance to the solution sample affects the increase in the output voltage until it reaches a maximum output voltage of $176.8 \mathrm{mV}$ at a distance of $650 \mu \mathrm{m}$. The fourth sample with a concentration of $150 \mathrm{ppm}$ minimum output voltage starts at $165.2 \mathrm{mV}$ and increases in voltage by increasing the distance to the solution sample until it reaches a maximum output voltage of $167.5 \mathrm{mV}$ at a distance of $700 \mu \mathrm{m}$.

The concentration of cholesterol solution $200 \mathrm{ppm}$ the output voltage starts at $157.1 \mathrm{mV}$ at a minimum distance. Increasing the distance to the solution sample affects the increase in the output voltage value until it reaches the maximum output voltage of $158.6 \mathrm{mV}$ at a distance of $700 \mu \mathrm{m}$. After reaching the maximum output voltage peak with increasing distance to the solution sample, the output voltage decreases the voltage value.

The sixth sample with a concentration of $250 \mathrm{ppm}$ minimum output voltage starts at $149.0 \mathrm{mV}$ and increases in voltage by increasing the distance to the solution sample until it reaches a maximum output voltage of $150.1 \mathrm{mV}$ at a distance of $750 \mu \mathrm{m}$.
The seventh sample concentration of 300 ppm output voltage starts $144.3 \mathrm{mV}$ at a minimum distance. Increasing the distance to the solution sample affects the increase in the output voltage until it reaches the maximum output voltage of $146.3 \mathrm{mV}$ at a distance of $750 \mu \mathrm{m}$. Of the seven samples reaching the maximum output voltage peak with increasing distance to the solution sample, the output voltage decreases the voltage value exponentially.

The output power profile of the experiment from a sample of seven variations in cholesterol concentration using a Gaussian quasi beam shows that the optical fiber output power will increase with increasing distance of the object shift from the optical fiber bundle. This can be indicated by an increase in voltage until it reaches the maximum voltage. After reaching the peak output power will decrease exponentially with increasing distance. The maximum voltage of each sample decreases with increasing cholesterol concentration caused by energy uptake. With the increase in the concentration of the solution from the sample used, the greater the energy absorption which causes the maximum voltage to decrease.

Figure 3 show the results of peak stress analysis on variations in cholesterol concentration using OFS flat mirror reflecting field showed a sensitivity of $0.21 \mathrm{mV} / \mathrm{ppm}$ and linearity of $95.1 \%$. The results of this sensitivity indicate that an approach to sensitivity values uses a Quasi Gaussian beam mathematically compared to sensitivity using a Gaussian beam. The results of the data analysis determined the cholesterol concentration using this flat mirror in accordance with the theoretical analysis obtained.

\section{CONCLUSION}

The conclusion that can be stated based on the results and discussion of this study is the output power profile of the experimental results using Quasi Gaussian beam shows that the optical fiber output power will increase by increasing the object's shift from optical fiber, after reaching the peak output power will decrease exponentially with increasing distance. The performance of the shift sensor of the Quasi Gaussian beam optical fiber to determine the concentration of cholesterol achieves high sensitivity, linearity and accuracy. Elemental sensing experiment validation can be used to determine the output voltage value (maximum / minimum) as a concentration function based on shift profile between fiber optic probes and cholesterol samples. Shifting the tip of the fiber optic to the reflecting plane can receive the maximum reflected laser beam by the fiber receiving and produce higher efficiency.

\section{ACKNOWLEDGMENT}

The authors would like to acknowledge The Airlangga University for providing Optical Laboratories. We also thank for Y. G. Yhun Yhuwana who has helped and advice for this research.

\section{REFERENCES}

[1] Samian, A. H. Zaidan, and M. Yasin, "Detection of Rhodamine B levels in distilled water based on displacement sensor using fiber coupler and concave mirror," J. Optoelectron. Adv. Mater., vol. 18, no. 11-12, pp. 988-992, 2016. 
[2] M. Yasin et al., "Intensity based optical fiber sensors for calcium detection," Optoelectron. Adv. Mater. Rapid Commun., vol. 9, no. 9-10, pp. 1185-1189, 2015.

[3] N. Hida, N. Bidin, M. Abdullah, and M. Yasin, "Fiber optic displacement sensor for honey purity detection in distilled water Fiber optic displacement sensor for honey purity detection in distilled water," Optoelectron. Adv. Mater. - Rapid Commun., vol. 7, no. July-August, pp. 565-568, 2013.

[4] M. Yasin, H. Ahmad, K. Thambiratnam, A. A. Jasim, S. W. Phang, and S. W. Harun, "Design of multimode tapered fibre sensor for glucose detection," Optoelectron. Adv. Mater. Rapid Commun., 2013.

[5] H. A. Rahman, S. W. Harun, M. Yasin, and H. Ahmad, "Fiber-optic salinity sensor using fiber-optic displacement measurement with flat and concave mirror,” IEEE J. Sel. Top. Quantum Electron., 2012.

[6] J. A. B. Faria and J. A. Brandao Faria, "Modeling the Y-branched Optical Fiber Bundle Displacement Sensor Using a Quasi-Gaussian Beam Approach," Microw. Opt. Technol. Lett., 2000.

[7] M. Budiyanto, Suhariningsih, and M. Yasin, "Optical fiber displacement sensor using concave mirror for cholesterol detection," in 2017
International Seminar on Sensors, Instrumentation, Measurement and Metrology (ISSIMM), 2017, pp. 150-153.

[8] J. Jaime, G. Rangel, A. Muñoz-Bonilla, A. Mayoral, and P. Herrasti, "Magnetite as a platform material in the detection of glucose, ethanol and cholesterol," Sensors Actuators, B Chem., 2017.

[9] A. N. Kozitsina, A. V. Okhokhonin, and A. I. Matern, "Amperometric detection of cholesterol using cobalt (II) chloride as an electrocatalyst in aprotic media," J. Electroanal. Chem., 2016.

[10] S. Nantaphol, O. Chailapakul, and W. Siangproh, "Sensitive and selective electrochemical sensor using silver nanoparticles modified glassy carbon electrode for determination of cholesterol in bovine serum," Sensors Actuators, B Chem., 2015.

[11] J. Ji, Z. Zhou, X. Zhao, J. Sun, and X. Sun, "Electrochemical sensor based on molecularly imprinted film at Au nanoparticles-carbon nanotubes modified electrode for determination of cholesterol," Biosens. Bioelectron., 2015.

[12] M. Budiyanto, Suhariningsih, and M. Yasin, "Cholesterol detection using optical fiber sensor based on intensity modulation," J. Phys. Conf. Ser., vol. 853, no. 1, 2017. 